\title{
Morphological classification and changes in dementia (Review)
}

\author{
ALEXANDRA TUDOR $^{1}$, ANTONIA IOANA VASILE $^{2 *}$, SIMONA CORINA TRIFU $^{3 *}$ and MIHAI BOGDAN CRISTEA ${ }^{4 *}$ \\ ${ }^{1}$ Department of Psychiatry, 'Prof. Dr. Alex. Obregia' Clinical Hospital of Psychiatry, 041914 Bucharest; \\ ${ }^{2}$ Department of General Medicine, Medical Military Institute, 010919 Bucharest; Departments of ${ }^{3}$ Clinical Neurosciences \\ and ${ }^{4}$ Morphological Sciences, 'Carol Davila' University of Medicine and Pharmacy, 020021 Bucharest, Romania
}

Received June 25, 2021; Accepted July 27, 2021

DOI: 10.3892/etm.2021.10955

\begin{abstract}
The progressive functional decline that involves both cognitive and neuropsychiatric symptoms characteristic to dementia is one of the leading research topics. The risk for dementia is an intertwined mix between aging, genetic risk factors, and environmental influences. APOE\&4, which is one of the apolipoprotein E (APOE) alleles, is the major genetic risk factor for late-onset of the most common form of dementia, Alzheimer's. Advances in machine learning have led to the development of artificial intelligence (AI) algorithms to help diagnose dementia by magnetic resonance imaging (MRI) in order to detect it in the preclinical stage. The basis of the determinations starts from the morphometry of cerebral atrophies. The present review focused on MRI techniques which are a leading tool in identifying cortical atrophy, white matter dysfunctionalities, cerebral vessel quality (as a factor for cognitive impairment) and metabolic asymmetries. In addition, a brief overview of Alzheimer's disease was presented and recent neuroimaging in the field of dementia with an emphasis on structural MR imaging and more powerful methods such as diffusion tensor imaging, quantitative susceptibility mapping, and magnetic transfer imaging were explored in order to propose a simple systematic approach for the diagnosis and treatment of dementia.
\end{abstract}

\section{Contents}

1. Introduction

2. Research methods

3. References to dementia medication

Correspondence to: Dr Antonia Ioana Vasile, Department of General Medicine, Medical Military Institute, 3-5 Institutul Medico-Militar Street, 010919 Bucharest, Romania

E-mail: antoniaioana97.vasile@gmail.com

${ }^{*}$ Contributed equally

Key words: dementia, antidepressant, depression, neuroplasticity, white matter, hippocampus
4. Elucidation of Parkinson's dementia and Huntington's dementia

5. Anatomically functional references to the importance of the hippocampus in dementia

6. Conclusion

\section{Introduction}

Dementia is not a specific disease; it affects each patient in a different way. It is a general term for the declining cognitive abilities with symptoms such as: losing track of the time, forgetfulness and becoming lost in familiar places.

From the etiology point of view, numerous causes are described including genetic factors, biological vulnerability, cerebral vascular vulnerability and cognitive, psychological and social vulnerability $(1,2)$.

Biological susceptibility is dependent on the following factors including i) preservation of the neuro-biochemical coefficient of inter-neuronal transmission, being described as the vulnerability of the acetylcholine (Ach) system and Tau proteins in Alzheimer's and Lewy body diseases; the vulnerability of the dopaminergic system in Parkinson's and front-temporal diseases; and the glutamatergic vulnerability in vascular dementia (3); ii) preservation of the neuron-astroglia functional ratio: deficiencies and massive neuronal losses in cortical dementias; and massive astrocyte deficiencies and losses in subcortical dementias; and iii) preservation of the connective relationship between different brain structures: temporo-parietal dementia in Alzheimer's dementia; front-temporal dementia in Pick's disease; cortical dementia with altered nucleus basalis: Dementia with Lewy bodies; dementia with altered nucleus basalis and secondary cortical deficiency: Dementia from Parkinson's disease and Huntington's dementia $(4,5)$.

Cerebral vascular vulnerability is a background of any dementia disorder being dependent on somatic conditioning (cardiovascular and metabolic diseases). Vasomotor effectors control cerebral perfusion pressure, which leads to self-regulation of cerebral blood flow (2).

Cerebral ischemia can occur as a result of reduced cerebral blood flow, which is due to impaired self-regulation. Most types of vascular dementia are based on cerebral ischemia. It is also assumed that this is a determining factor in the pathology of Alzheimer's disease, intensifying its clinical manifestations; 
thus, a decrease in cerebral blood flow has been identified in Alzheimer's patients correlated with a degree of cerebrovascular pathology. In the case of cognitive, psychological and social vulnerability, the premorbid personality determines the clinical profile of the non-cognitive manifestations that can precipitate the evolution (2).

\section{Research methods}

The present review includes the most recent and significant articles concerning morphological changes in dementia presented in a structured way. The last ten years of research (2010-2020) were targeted but with reference to older studies if deemed important to our topic. The relevance of the selected articles is given by the number of subjects involved, by the variety of research methods, by the differences between patients. In recent years, most research methods have been based on magnetic resonance imaging (MRI).

Elderly patients admitted to psychiatric hospitals have multiple somatic comorbidities, which should alert specialists to the importance of selecting optimal psychotropic drugs which are effective but with minimal risk of pleural adverse reactions. The risk of stroke, hemorrhagic or ischemic, increases with age and depends on the presence of risk factors (6). In numerous cases, until the time of admission to a psychiatric ward, neither the patient nor the family was aware of the presence of minor ischemic strokes in the past, and minor cognitive impairments were often compensated by the intellect. Although the literature differentiates vascular dementia from Alzheimer's, clinical practice highlights an increased number of mixed dementias. When there is associated vascular pathology or alcoholism as a comorbidity, the decline in cognitive function is more abrupt, with the onset of higher-grade dementia on the Reisberg scale (7). There are also reverse clinical situations, in which a subclinical Alzheimer's disease was first present, over which an ischemic stroke occurred, this being the moment when the severity of cognitive impairment was revealed. The more the infarction areas and the more they affect the limbic region, the medial region, the frontal cortex and the white matter, the more the intellect collapses. When there are cerebrovascular lesions, the density of neurofibrillary plaques in the temporal and frontal isocortex decreases. The correlation with impairment of cognitive function and the severity of dementia is lower if microinfarcts occur in the hippocampal region. A study of 186 Alzheimer's patients and 13 subjects without cognitive impairment revealed that in patients with ischemic or hemorrhagic stroke or those with Parkinson's disease, neurofibrillary tangles have lower density (8).

In psychiatric practice mixed dementias that occur on chronic ischemic brain lesions are frequently encountered, which in turn are caused by changes in the production of factors involved in vasoregulation [endothelin 1 (ET1) and angiotensin converting enzyme (ACE)] (8). In addition, also important are factors involved in angiogenesis, such as: vascular endothelial growth factor (VEGF), which can provide an indication of the self-repair capacity of the brain, the potential for brain neuroplasticity, the restoration of functional circuits involved in maintaining attention, memory and cognition (8).
A previous study with 30 autopsies was performed on subjects aged 34-91 years, of which 12 were men (40\%) and 18 were women $(60 \%)$. All subjects had cardiovascular and cerebrovascular comorbidities, but the definite diagnosis of vascular dementia was established only for 10 cases. The conclusion of the study was that, with increasing age, the number of neurons in the frontal cortex significantly decreases for groups aged 71-80, 81-90 and 91-100 years old, compared with groups aged 31-40 and 41-50 years old. The percentage differences reached $13.78 \%$ (9). Studying the 10 cases in which the diagnosis of vascular dementia was certain, the autopsy on the frontal lobes revealed the loss of axons, a high process of gliosis instead of lost axons, the expansion of perivascular spaces, the profile suggesting, in one word, the presence of leukoaraiosis (9). In $51 \%$ of cases, the cerebral blood vessels revealed signs of atherosclerosis, these lesions reaching up to $50 \%$ of the surface, which determines the intellectual impairment of higher cognitive functions (anticipation, planning, sequencing, operationalization, control, ability to operate on the abstract, the use of hypothetical-deductive reasoning and judgments) (9). As markedly as the signs of atherosclerosis are present at the level of the frontal lobes, it can be expected that at the behavioral level, violent pathologies, aggression, disinhibition, inability to anticipate the consequences of the patient's actions are exhibited, clinically being referred to "moriatic syndrome' (10).

Leukoaraiosis is highlighted in MRI by hyperintensity in the cerebral white matter, while on computed tomography (CT) a decrease in scanning intensity is observed. Leukoaraiosis observed by imaging has been revealed to be correlated with clinical dementia (11). From a pathophysiological point of view, arteriosclerotic mechanisms in small blood vessels are important, where there is an increased risk of heart attack. This happens at the level of white matter, and hypertension predisposes. In cerebral amyloid angiopathy, the interstitial fluid in the white matter is no longer properly eliminated in order to be taken up by the blood vessels; the pathophysiological mechanism in question risking to cause infarction (11). This mechanism is all the more present, and has a pathological significance the greater the age of the subject (12). The drainage of interstitial fluid from an aged brain is being discussed, over which amyloid angiopathy overlaps, these being the primary mechanisms in the pathogenesis of dementia. Consequently, psychopharmacology has focused on the discovery of new therapeutic classes that facilitate the efficient drainage of interstitial fluid, for a proper elimination of soluble metabolites (13). Although the option for clozapine is surprising, it is beneficial in such patients (14), as clozapine does not in itself act on the drainage of interstitial fluid, but is a facilitator that allows improved efficacy of acetylcholinesterase inhibitors. Clozapine is thus retained as a backup medication when resistance to antidementia treatment appears to have set in. The tau protein is located in the inner body of neurons and is part of the microtubule that provides intracellular transport of nutrients as well as ions. Brain stabilization depends on the integrity of tau proteins (because they regulate microtubule stability) and microtubules, regardless of age $(15,16)$. In Alzheimer's disease and fronto-temporal dementia, dystrophic neuritis tau-immunoreactive argilophiles and neurofibrillary tangles (NFTs) in the neocortex are identified. The greater 
their presence, the more significant is the neuronal pathology and cognitive function impairment (17). A previous study reported that aluminum in certain foods is a risk factor that generates, maintains and potentiates Alzheimer's disease. Moreover, it is known that exposure to aluminum in excess, even if it is caused by every day work, causes inflammatory brain activity (18).

A cohort study was performed in which the brains of 141 subjects with and without dementia were autopsied. Among the conclusions, it was noted: at the time of death, $>50 \%$ of the elderly had anatomical structural changes that indicated cognitive impairment; most of the deceased presented as mixed pathology ischemic strokes that occurred over Alzheimer's disease. The more somatic comorbidities the patient has, the higher the chances of developing a dementia pathology, compared with the situation in which the subject would have a single comorbidity (19).

Structural imaging data obtained by MRI indicated biomarkers such as scalar values: the volume of a structure and the rate of morphological variation of it being indicators of a presymptomatic evolution (20). In Alzheimer's disease, subcortical structures are not subject to symmetrical neurodegeneration (21). For example, the hippocampus acquires a significant longitudinal asymmetry, as well as the amygdala; the asymmetry in question developing progressively with the severity of the disease, and often the asymmetry being the one that can be identified before the clinical evidence of cognitive deficit. For this reason, neuroimaging studies in which specialists investigate longitudinal asymmetry in the hippocampus, amygdala, caudate nucleus and cortex become predictive, either to highlight the onset of Alzheimer's disease or to identify when to change the diagnosis from mild cognitive impairment to stage IV Alzheimer's dementia (22). In the past, positron emission tomography (PET) with ${ }^{18} \mathrm{~F}$-fluorodeoxyglucose was used to identify significant metabolic asymmetries between the right and left regions in the frontal and parietal cortex, respectively. These were relevant in mild Alzheimer's disease. A 2011 PET study used the compound ${ }^{11} \mathrm{C}$-Pittsburgh B, when it is required to emphasize the asymmetric spatial distribution of amyloid- $\beta$ and the positive correlation between amyloid- $\beta$ deposition and asymmetry; the mechanism involved being hypometabolic. As Alzheimer's disease progresses, right-to-left asymmetries remain stable in terms of targeting and become more pronounced over time (23).

Amyloid- $\beta$ loading asymmetries, hypometabolism, lateralization of the pathology, neurodegeneration, are not only present at the onset of the disease, but remain a feature in all Reisberg stages of dementia, although there are cases where cognitive impairment is not as severe as asymmetry and the rest of the histopathological data (23). In Alzheimer's patients, MRI captures how cortical atrophy occurs early and progresses faster in the left hemisphere than in the right hemisphere (24). In imaging studies, SPECT with ${ }^{133} \mathrm{Xe}$ is also used, which indicates an absolute measure of regional cerebral blood flow (rCBF) and technetium-99m-hexamethylpropyleneamineoxime (99mTc-HMPAO), with the images provided being of high resolving quality (25). With this technique, 47 patients with fronto-temporal dementia were analyzed, the images were evaluated by two neuroimaging. Regarding the temporal lobe variant, 10 of the patients with fronto-temporal dementia presented a variant of the temporal lobe (TLV), a fact confirmed by consensus by both neuroimaging. This subgroup of patients with TLV had a significant bitemporal and orbitofrontal asymmetry, but a smaller asymmetry in the frontal cingulate, dorso-lateral and polar areas (25). The two neuroimaging had the task of detecting patients in whom the predominant variant of the temporal lobe with predominant right asymmetry or with predominant left asymmetry (RTLV or LTLV) predominates. However, all subjects with TLV had orbito-frontal hypoperfusion and had bilateral temporal involvement (25). The clinical division between RTLV and LTLV was based on hypoperfusion asymmetry. The ultimate goal of the study was to highlight the link between temporal dysfunction and behavioral changes in these patients. These 10 subjects with TLV were compared with the remaining 37 subjects of the study, who had selective hypoperfusion at the frontal level, not at the temporal level. Of the remaining 37 subjects, 10 of them had diffuse hypoperfusion in the frontal lobes, while in the temporal lobes the diffusion of cerebral blood flow was normal. Of these 10 subjects with frontal hypoperfusion, 5 of them had hypoperfusion on the right side, and in the other 5 cases, on the left side (25).

Another study was performed on 93 subjects with mixed dementia (Alzheimer's disease doubled by subcortical cerebrovascular disease). Patients were followed longitudinally and MRI images were analyzed to measure the following volumes: cortical gray matter, hippocampus, white matter hyperintensities, gaps. Pathological correlations were identified between decreased cortical gray matter volume, Alzheimer's disease, subcortical vascular pathology and arteriosclerotic phenomenon (26). Decreased hippocampal volume and atrophies at this level correlate with Alzheimer's disease. The hyperintensities identified in the white matter, the number of gaps and subcortical vascular pathology correlate directly with aging (24). In other words, the presence of gaps and changes in the white matter are pathognomonic of vascular disease. The neuropathological basis of aging is cortical and hippocampal atrophy, but dementia is a much more complex clinical phenomenon, in which multiple similar brain structures mediate cognitive decline (24).

\section{References to dementia medication}

The issue of dementia highlights a dysfunction of the cholinergic system, which has as a clinical resonance the memory disorder, especially short-term memory (27). The most effective way to improve the cholinergic system in Alzheimer's has been revealed to be the inhibition of acetylcholine metabolism by blocking the enzyme acetylcholinesterase (AchE). Thus, more acetylcholine remains available in the synaptic cleft, which improves memory and slows the progression of the disease. All antidementia drugs do not act on already destroyed neurons, but increase synaptic transmission on the proportion of neurons still intact. Acetylcholinesterase inhibitors have a dual mechanism of action in addition to performing a positive allosteric modulation on postsynaptic nicotinic receptors.

Another hypothesis issued in Alzheimer's disease refers to excess glutamate, which contributes to the destruction of neurons by excitotoxicity (28). 
Medication used to treat dementia includes donepezil (acetylcholinesterase inhibitor: 5-10 mg/day); galantamine (acetylcholinesterase inhibitor and nicotinic receptor modulator: $12-24 \mathrm{mg} /$ day); rivastigmine (acetylcholinesterase and butyryl cholinesterase inhibitor: 6-12 mg/day); and memantine (rNMDA antagonist: 10-20 mg/day) (29).

Serum cholinesterase inhibitors (ChE) are the mainstay in the treatment of Alzheimer's disease (29) for the following reasons: i) they increase cholinergic transmission, inhibiting the action of cholinesterase in the synaptic cleft; ii) they require relatively intact cholinergic postsynaptic receptors to benefit from increased acetylcholine levels; iii) they are useful in the treatment of mild to moderate dementia [Mini-Mental State Examination (MMSE) score 10-26]. Most studies demonstrate their efficacy in Alzheimer's dementia, but there are data that support the positive effects for both mixed dementia and dementia from Parkinson's disease with a slight improvement of cognition in daily activities and possibly effective in advanced forms, but the data are less clear (30-32).

The side effects of acetylcholinesterase inhibitors are: cholinergic side effects including diarrhea, nausea and vomiting which are generally transient and mild (20\%); bradycardia and syncope, as potential side effects; as well as headache and dizziness (33).

With regard to memantine it can be said that it is a weak to moderate antagonist of N-methyl-D-aspartate (NMDA) glutamate receptor, which decreases normal receptor activity if there is an excess of glutamate (34); with minimum hepatic metabolism on CYP450, and instead requires dose reduction in patients with kidney disease. It exhibits moderate-severe antidementia efficacy; fewer side effects compared with AchI (most often it can cause dizziness). As a partial agonist of glutamate receptors, it has been used in Germany for over 20 years, not only in cognitive disorders, but also in various other neurological symptoms (35).

Rivastigmine (4.6-13.3 mg) has an inhibitory action on both acetylcholinesterase and butyryl cholinesterase (36).

Galantamine is an allosteric modulator of nicotinic acetylcholine receptors also with renal elimination, effective in vascular dementia, in Lewy bodies (37).

Memantine and cholinesterase inhibitors remain the main drugs of treatment, but over time other agents have been considered, without much success including Ginkgo biloba, non-steroidal anti-inflammatory drugs, estrogen, selegiline and vitamin $\mathrm{E}(38-40)$.

\section{Elucidation of Parkinson's dementia and Huntigton's dementia}

Parkinson's disease is a multifactorial and progressive disorder, of neurological and chronic nature, which is based on the selective loss of dopaminergic neurons from the substantia nigra pars compacta (41). Over time, motor manifestations of Parkinson's disease (tremor and stiffness) associated with cognitive impairment, can progress to dementia. At the neuronal level, intracellular inclusions (Lewy bodies) are formed, which contain $\alpha$-synuclein aggregates. Parkinson's disease involves symptoms of neuritis in the brainstem, damage to the limbic system and cortical areas. Amyloid- $\beta$ deposits are also formed. Dopaminergic neurons degenerate, which leads to a decrease in dopamine in the synaptic cleft, a phenomenon which in turn slows down dopaminergic neurotransmission related to motor circuits. The mechanism occurs mainly in the basal ganglia. Gastrointestinal tract dysfunctions are associated (42), deteriorating phenomena that can lead to dementia, and from a psychopathological point of view, a whole plethora of manifestations, from sleep disorders to depression, symptoms that have been found in recent years, which improve considerably under the application of low frequency repetitive transcranial magnetic stimulation (rTMS). Dopaminergic neurons in the substantia nigra pars compacta deteriorate due to monoamine oxidase, which causes an aberrant metabolism of dopamine and causes the accumulation of hydrogen peroxide, which is toxic (43).

When Parkinson's disease develops with dementia, decreased judgment, thinking, and critical thinking occur after at least one year and are the consequence of aberrant microscopic deposits of $\alpha$-synuclein. This compound is a protein that exists on a large scale in the brain, with a function not fully known (44), but in patients with Parkinson's disease is found in excess, promoting the development of deposits called Lewy bodies, revealed in 50-80\% of those with Parkinson's, their number increasing as the disease progresses (45). Old age, severity of motor symptoms, cognitive impairment, even if it is mild, but present from the beginning, are serious factors in Parkinson's disease. Excessive daytime sleepiness, postural instability and gait disorders (PIGD) and, especially, the presence of hallucinations, without other signs of dementia, are factors of poor prognosis. To these are added: disorders of concentration, stability and selectivity of attention, changes in memory function, both working and long-term, both fixation and evocation, impaired reasoning, hypothetical-deductive inferences and judgment (46). Positive psychiatric symptoms may overlap, beginning with erroneous processing and consequent interpretation of visual information, leading to illusions and hallucinations, along with ideas of prevalent or delusional intensity, paranoid spectrum (frequent being those of persecution, pursuit or jealousy, to which the patient is not critical and which is associated with behaviors of significant aggression). Among the sleeping disorders, pathognomonic are those with rapid movements of the eyeballs (47). Usually, there is approximately a year between the onset of motor symptoms in Parkinson's disease and the onset of symptoms of cognitive impairment. There are no treatments to stop or slow down neuronal damage in Parkinson's. Cholinesterase inhibitors used for the cognitive sphere in Alzheimer's can also help in Parkinson's dementia, but through an eminently symptomatic action. This category of drugs favorably influences visual perceptual disorders, sleep changes, prevalent and delusional distortions in thinking and violent behavioral pathology, inappropriate to the context $(29,30)$. However, there are reported cases of hallucinations or confusion in patients with Parkinson's dementia. Selective serotonin reuptake inhibitors, used to treat comorbid depression, melatonin or clonazepam, are sometimes useful to treat REM sleep disorders (48). Clonazepam is also useful as a muscle relaxant.

Movement disorders are caused by defects in the functioning of the basal ganglia, while dementia is the result of cortical abnormalities. In other words, the discussion is about two different disorders, at the motor level and at the cognitive 
level, which involve different anatomical areas (49). However, new research sheds light on these divergences, as the basal ganglia have rich connections to the cerebral cortex, especially the prefrontal area, through the thalamus. The caudate nucleus and the thalamus are important areas in the mechanisms of intellectualization, rationalization and in the process of knowledge. There is a wide network that starts at the prefrontal cortex, involves the caudate nucleus and the dorso-medial thalamus and is of interest to both attention and movement. The network in question gives human behavior a sense of personality, supporting the general filter of attention and integrating thinking with emotion and motivation. Considering this theory of the existence of an extensive network, even in situations where the frontal lobe is not pathologically involved, but only the subcortical structures are affected, in terms of clinical expression, the patient in question behaves as if he had a 'moriatic' pathology.

There are also biochemical explanations in addition to the anatomical ones. Although in Parkinson's disease the dopaminergic system is the main system affected, there are also disorders in the neurotransmitters acetylcholine and serotonin, which explains the cognitive, affective and movement pathology (50).

Huntington's disease is also a genetic-based neurological disorder (a mutation in the huntingtin gene on chromosome 4). This is an excess repetition of the CAG sequence of trinucleotides. Therefore, a mutant huntingtin protein can form abnormal aggregates, which abnormally regulates transcription, modifies the homeostasis of neuronal membrane proteins, and multiple aggregates will develop that inhibit intra- and intercellular exchanges, by affecting intracytoplasmic vesicles. From the terminal button of the presynaptic axon to the dendrite of the postsynaptic neuron, the axonal traffic of neurotransmitters suffers. Synaptic plasticity is low and glial cells cannot be compensated either $(51,52)$.

Huntington's disease affects movement, mood and thinking. The distinguishing symptom is an uncontrolled movement of the arms, legs, head and face (mainly the upper half of the body). Symptoms develop between the ages of 30 and 50. At the knowledge level, the skills of abstract thinking, judgment and reasoning, perceiving the absurd, organizing, planning, sequencing and providing retroactive feedback decrease. Concurrently, the concentration of attention and all three forms of memory are disturbed. Affectively, mood swings are observed, ranging from depression and apathy, to anxiety, anger, irritability and irascibility. The pathognomonic feature is the obsessive-compulsive behavior. It can take the form of perseverance in asking a certain question or in the adhesiveness and psychic viscosity of a certain idea, theme, activity or individual (53).

A study of subjects likely to develop Huntington's disease would reveal early decreases in processing speed, the slowing of cognition being a foremost manifestation of this disorder (53). This is the distinguishing feature of Huntington's dementia, compared with Alzheimer's dementia, in which the first psychopathological change that occurs is memory loss. In Huntington's disease, the decline in memory occurs relatively late, but it is an unfavorable predictor of disease progression in terms of loss of functionality. Memory deficiencies in Huntington's disease are due to the inability to systematically and successfully retrieve stored information. In other words, the process of evocation is the one affected (both recognition and recollection). The slowness of the processing speed correlates with the difficulties in mnemonic evocation and is related to the frontal lobes (54). The results of a study by Rohrer et al indicated that in Huntington's disease, poor memory performance also affects motor recovery, as the loss of the ability to store information appears to be related to the maintenance of motor skills (55).

\section{Anatomically functional references to the importance of the hippocampus in dementia}

Before the discovery of Alzheimer's disease, there was discussion of hyperactivity in the memory network, which was considered to consist of the hippocampus, medial temporal lobe and several cortical regions. Recently, this hyperactivity in mnemonic function is considered to be due to neuronal excitotoxicity (56).

The hippocampus consists of three distinct regions: the dentate gyrus, cornu ammonis 3 (CA3) and cornu ammonis 1 (CA1) (57). The influx of blood from the CA3, the second layer of the entorhinal area, reaches the dentate gyrus through the perforating pathway, where the motor fibers circulate. Schaffer collateral neurons are also involved, which have an effect on the efficiency and duration of storage memory, in which the serotonergic neurotransmitter also plays an important role. Through them, the information goes from the CA1 to the CA3. If the synaptic connections between the entorhinal area and the dentate gyrus decrease at the level of the perforating pathway, the memory will be severely affected (57). If the signals from the entorhinal area decrease, the rate of pulse transmission at the CA3 level will increase. If the memory deficiency is due to a problem at the level of CA1, it is identified as the clinical situation in which the number of silent synapses has increased, i.e. those synapses that lack the excitatory glutamatergic AMPA receptors. Concurrently, the number of calcium channels increases, which in the long run will give excitatory signals, which explains why CA1 neurons are more sensitive to excitotoxicity and, consequently, increase the risk of developing Alzheimer's disease (57). Research on laboratory mouse have revealed that neuronal hyperactivity increases amyloid- $\beta$ production (58). The increased activity of neurons in CA1 causes the formation of amyloid- $\beta$ aggregates (58).

Atrophies in the hippocampus and entorhinal cortex play an important role in memory impairment in the early stages of Alzheimer's disease (59). These two aforementioned structures, together with the amygdala, become severely atrophied in the late stages of the disease (VI/VII Reisberg). At the level of the hippocampus, neuritic plaques are formed, these being collections of neurites around a central amyloid nucleus. Adjacent, intracytoplasmic bundles of hyperphosphorylated protein filaments are revealed. These 'windings' move the nucleus of the neuron. Mainly, neuritic plaques have their favorite place of formation in the hippocampus. Furthermore, in the hippocampus, there is granulovacuolar degeneration, small cytoplasmic vacuoles are formed, each of which has an argyrophilic granule inside (60). In addition, in the hippocampus, in the pyramidal cells, the Hirano bodies are formed. These are inclusions of eosinophils, composed mainly of actin filaments (61). 
Other research suggests that the reduction in insulin rate and insulin growth factors, which are identified in neurons (IGF), also contribute to neuronal degeneration in Alzheimer's disease. Post-mortem studies have revealed these anatomopathological records at the hippocampal level, as well as in the frontal lobes and thalamus. The cerebellum is not affected by Alzheimer's disease and, as a result, insulin and insulin growth factor are normal at this level (62-64).

\section{Conclusions}

The order in the frequency of dementias in clinical pathology, is revealed to be: Alzheimer's disease, followed by vascular dementia, dementia with Lewy bodies and, less frequently, fronto-temporal dementia $(33,34)$.

According to studies that have been reviewed, the highest frequency is found among patients with mixed lesions and among these, the most common are those associated with Alzheimer's disease with lacunar cerebral infarct, caused by either ischemic stroke or thrombi which have migrated from other areas such as the carotid arteries or heart. When several pathologies (predominantly cardiovascular) coexist at the same time and potentiate each other, there is a threefold increase in the chances of developing dementia. The favorable response to cholinesterase inhibitors should be noted.

The confirmed MRI and histopathological asymmetry between the right and left hemispheres should also be noted, an observation that appeared early in the evolution of the disease and it is progressively accentuated, more for the left hemisphere than for the right one.

Last but not least, the theory of poor interstitial fluid drainage is worth mentioning, especially with regard to an aged brain. This is potentiated by amyloid angiopathy, and this is a significant factor in the pathogenesis of dementia.

\section{Acknowledgements}

Not applicable.

\section{Funding}

No funding was received.

\section{Availability of data and materials}

Not applicable.

\section{Authors' contributions}

SCT and AT designed and drafted the initial review. AT and AIV gathered the medical information, established the structure of the article, investigated the present topic of research and gathered the important information. SCT and AIV confirm the authenticity of all the raw data. SCT and MBC finalized the work and approved the final version of the work. All authors read and approved the final manuscript.

\section{Ethics approval and consent to participate}

Not applicable.

\section{Patient consent for publication}

Not applicable.

\section{Competing interests}

The authors declare that they have no competing interests.

\section{References}

1. Sacuiu SF: Dementias. Handb Clin Neurol 138: 123-151, 2016.

2. Raz L, Knoefel J and Bhaskar K: The neuropathology and cerebrovascular mechanisms of dementia. J Cereb Blood Flow Metab 36: 172-186, 2016.

3. Takeda S: Progression of Alzheimer's disease, tau propagation, and its modifiable risk factors. Neurosci Res 141: 36-42, 2019.

4. Kertesz A: Frontotemporal dementia, Pick's disease. Ideggyogy Sz 63: 4-12, 2010.

5. Gomperts SN: Lewy body dementias: Dementia with Lewy bodies and Parkinson disease Dementia. Continuum (Minneap Minn) 22 (2 Dementia): 435-463, 2016.

6. Wachinger C, Salat DH, Weiner M and Reuter M; Alzheimer's Disease Neuroimaging Initiative: Whole-brain analysis reveals increased neuroanatomical asymmetries in dementia for hippocampus and amygdala. Brain 139: 3253-3266, 2016.

7. Trifu S, Carp EG and Nadoleanu A: Alcohol as a substitute, mask of depression and 'antidote' of narcissism. Eur Proc Soc Behav Sci 31: 986-994, 2017.

8. Barker R, Ashby EL, Wellington D, Barrow VM, Palmer JC, Kehoe PG, Esiri MM and Love S: Pathophysiology of white matter perfusion in Alzheimer's disease and vascular dementia. Brain 137: 1524-1532, 2014.

9. Kolomiytsev AK and Bazilevich AV: Morphologic changes in vascular dementia. Adv Stud Biol 9: 101-104, 2017.

10. Trifu SC, Tudor A and Radulescu I: Aggressive behavior in psychiatric patients in relation to hormonal imbalance (Review). Exp Ther Med 20: 3483-3487, 2020.

11. Weller RO, Hawkes CA, Kalaria RN, Werring DJ and Carare RO: White matter changes in dementia: Role of impaired drainage of interstitial fluid. Brain Pathol 25: 63-78, 2015.

12. Kalaria RN: Small vessel disease and Alzheimer's dementia: Pathological considerations. Cerebrovasc Dis 13 (Suppl 2): S48-S52, 2002.

13. Jellinger KA and Attems J: Neuropathological evaluation of mixed dementia. J Neurol Sci 257: 80-87, 2007.

14. Dragoi AM, Radulescu I, Năsui BA, Pop AL, Varlas VN and Trifu S: Clozapine: An updated overview of pharmacogenetic biomarkers, risks, and safety-particularities in the context of COVID-19. Brain Sci 10: 840, 2020.

15. Johnson GV and Stoothoff WH: Tau phosphorylation in neuronal cell function and dysfunction. J Cell Sci 117: 5721-5729, 2004.

16. Silva MC and Haggarty SJ: Tauopathies: Deciphering disease mechanisms to develop effective therapies. Int J Mol Sci 21: 8948,2020

17. Kalaria RN, Kenny RA, Ballard CG, Perry R, Ince P and Polvikoski T: Towards defining the neuropathological substrates of vascular dementia. J Neurol Sci 226: 75-80, 2004.

18. Bondy SC: Low levels of aluminum can lead to behavioral and morphological changes associated with Alzheimer's disease and age-related neurodegeneration. Neurotoxicology 52: 222-229, 2016.

19. Schneider JA, Arvanitakis Z, Bang W and Bennett DA: Mixed brain pathologies account for most dementia cases in community-dwelling older persons. Neurology 69: 2197-2204, 2007.

20. O'Brien JT and Thomas A: Vascular dementia. Lancet 386: 1698-1706, 2015.

21. Jagust WJ, Zheng L, Harvey DJ, Mack WJ, Vinters HV, Weiner MW, Ellis WG, Zarow C, Mungas D, Reed BR, et al: Neuropathological basis of magnetic resonance images in aging and dementia. Ann Neurol 63: 72-80, 2008.

22. Schneider JA, Wilson RS, Bienias JL, Evans DA and Bennett DA: Cerebral infarctions and the likelihood of dementia from Alzheimer disease pathology. Neurology 62: 1148-1155, 2004.

23. Laforce R Jr and Rabinovici GD: Amyloid imaging in the differential diagnosis of dementia: Review and potential clinical applications. Alzheimers Res Ther 3: 31, 2011. 
24. Jack CR Jr, Petersen RC, Xu Y, O'Brien PC, Smith GE, Ivnik RJ, Boeve BF, Tangalos EG and Kokmen E: Rates of hippocampal atrophy correlate with change in clinical status in aging and AD. Neurology 55: 484-489, 2000.

25. Edwards-Lee T, Miller BL, Benson DF, Cummings JL, Russell GL, Boone K and Mena I: The temporal variant of frontotemporal dementia. Brain 120: 1027-1040, 1997.

26. Palesi F, De Rinaldis A, Vitali P, Castellazzi G, Casiraghi L, Germani G, Bernini S, Anzalone N, Ramusino MC, Denaro FM, et al: Specific patterns of white matter alterations help distinguishing Alzheimer's and vascular dementia. Front Neurosci 12: 274, 2018.

27. Hshieh TT, Fong TG, Marcantonio ER and Inouye SK: Cholinergic deficiency hypothesis in delirium: A synthesis of current evidence. J Gerontol A Biol Sci Med Sci 63: 764-772, 2008.

28. Revett TJ, Baker GB, Jhamandas J and Kar S: Glutamate system, amyloid $\beta$ peptides and tau protein: Functional interrelationships and relevance to Alzheimer disease pathology. J Psychiatry Neurosci 38: 6-23, 2013.

29. Grossberg GT: Cholinesterase inhibitors for the treatment of Alzheimer's disease: Getting on and staying on. Curr Ther Res Clin Exp 64: 216-235, 2003.

30. van Laar T, De Deyn PP, Aarsland D, Barone P and Galvin JE: Effects of cholinesterase inhibitors in Parkinson's disease dementia: A review of clinical data. CNS Neurosci Ther 17: 428-441, 2011.

31. Dyer SM, Laver K, Pond CD, Cumming RG, Whitehead C and Crotty M: Clinical practice guidelines and principles of care for people with dementia in Australia. Aust Fam Physician 45: 884-889, 2016

32. Gauthier S, Patterson C, Chertkow H, Gordon M, Herrmann N, Rockwood K, Rosa-Neto P and Soucy JP; CCCDTD4 participants: 4th canadian consensus conference on the diagnosis and treatment of dementia. Can J Neurol Sci 39 (Suppl 5): S1-S8, 2012 (In English, French).

33. Colović MB, Krstić DZ, Lazarević-Pašti TD, Bondžić AM and Vasić VM: Acetylcholinesterase inhibitors: Pharmacology and toxicology. Curr Neuropharmacol 11: 315-335, 2013.

34. McShane R, Westby MJ, Roberts E, Minakaran N, Schneider L, Farrimond LE, Maayan N, Ware J and Debarros J: Memantine for dementia. Cochrane Database Syst Rev 3: CD003154, 2019.

35. Overshott R and Burns A: Treatment of dementia. J Neurol Neurosurg Psychiatry 76 (Suppl 5): v53-v59, 2005.

36. Schneider LS, Mangialasche F, Andreasen N, Feldman H, Giacobini E, Jones R, Mantua V, Mecocci P, Pani L, Winblad B and Kivipelto M: Clinical trials and late-stage drug development for Alzheimer's disease: An appraisal from 1984 to 2014. J Intern Med 275: 251-283, 2014.

37. Lilienfeld S: Galantamine-a novel cholinergic drug with a unique dual mode of action for the treatment of patients with Alzheimer's disease. CNS Drug Rev 8: 159-176, 2002.

38. Hashiguchi M, Ohta Y, Shimizu M, Maruyama J and Mochizuki M: Meta-analysis of the efficacy and safety of Ginkgo biloba extract for the treatment of dementia. J Pharm Health Care Sci 1: 14, 2015.

39. Benito-León J, Contador I, Vega S, Villarejo-Galende A and Bermejo-Pareja F: Non-steroidal anti-inflammatory drugs use in older adults decreases risk of Alzheimer's disease mortality. PLoS One 14: e0222505, 2019.

40. Farina N, Llewellyn D, Isaac MG and Tabet N: Vitamin E for Alzheimer's dementia and mild cognitive impairment. Cochrane Database Syst Rev 1: CD002854, 2017.

41. Walker Z, Possin KL, Boeve BF and Aarsland D: Lewy body dementias. Lancet 386: 1683-1697, 2015.

42. Travagli RA, Browning KN and Camilleri M: Parkinson disease and the gut: New insights into pathogenesis and clinical relevance. Nat Rev Gastroenterol Hepatol 17: 673-685, 2020.
43. Trist BG, Hare DJ and Double KL: Oxidative stress in the aging substantia nigra and the etiology of Parkinson's disease. Aging Cell 18: e13031, 2019.

44. Rockenstein E, Nuber S, Overk CR, Ubhi K, Mante M, Patrick C, Adame A, Trejo-Morales M, Gerez J, Picotti P, et al: Accumulation of oligomer-prone $\alpha$-synuclein exacerbates synaptic and neuronal degeneration in vivo. Brain 137: 1496-1513, 2014.

45. Stefanis L: $\alpha$-Synuclein in Parkinson's disease. Cold Spring Harb Perspect Med 2: a009399, 2012.

46. Meireles J and Massano J: Cognitive impairment and dementia in Parkinson's disease: Clinical features, diagnosis, and management. Front Neurol 3: 88, 2012.

47. Jung I and Kim JS: Abnormal eye movements in parkinsonism and movement disorders. J Mov Disord 12: 1-13, 2019.

48. Wichniak A, Wierzbicka A, Walęcka M and Jernajczyk W: Effects of antidepressants on sleep. Curr Psychiatry Rep 19: 63, 2017.

49. Martilla RJ and Rinne UK: Dementia in Parkinson's disease. Arch Neurol Scand 54: 431-441, 1976.

50. Behari M, Bhattacharyya KB, Borgohain R, Das SK, Ghosh B, Kishore A, Krishnan S, Mridula KR, Muthane U, Pal PK, et al: Parkinson's disease. Ann Indian Acad Neurol 14 (Suppl 1): S2-S6, 2011.

51. Mitchell CT, Krier I, Arjomand J, Borowsky B, Tabrizi SJ and Leavitt BR; TRACK-HD Investigators Luthi-Carter R: Longitudinal expression changes are weak correlates of disease progression in Huntington's disease. Brain Commun 2: fcaa172, 2020.

52. Potkin KT and Potkin SG: New directions in therapeutics for Huntington disease. Future Neurol 13: 101-121, 2018.

53. Pla P, Orvoen S, Saudou F, David DJ and Humbert S: Mood disorders in Huntington's disease: From behavior to cellular and molecular mechanisms. Front Behav Neurosci 8: 135, 2014.

54. Butters N, Wolfe J, Granholm E and Martone M: An assessment of verbal recall, recognition and fluency abilities in patients with Huntington's disease. Cortex 22: 11-32, 1986.

55. Rohrer D, Salmon DP, Wixted JT and Paulsen JS: The disparate effects of Alzheimer's disease and Huntington's disease on semantic memory. Neuropsychology 13: 381-388, 1999.

56. Setti SE, Hunsberger HC and Reed MN: Alterations in hippocampal activity and Alzheimer's disease. Transl Issues Psychol Sci 3: 348-356, 2017.

57. Dalton MA,Zeidman P, Barry DN, Williams E and Maguire EA Segmenting subregions of the human hippocampus on structural magnetic resonance image scans: An illustrated tutorial. Brain Neurosci Adv 1: 2398212817701448, 2017.

58. Vyas Y, Montgomery JM and Cheyne JE: Hippocampal deficits in amyloid- $\beta$-related rodent models of Alzheimer's disease. Front Neurosci 14: 266, 2020.

59. deToledo-Morrell L, Stoub TR, Bulgakova M, Wilson RS, Bennett DA, Leurgans S, Wuu J and Turner DA: MRI-derived entorhinal volume is a good predictor of conversion from MCI to AD. Neurobiol Aging 25: 1197-1203, 2004

60. Köhler C: Granulovacuolar degeneration: A neurodegenerative change that accompanies tau pathology. Acta Neuropathol 132: 339-359, 2016.

61. Mitake S, Ojika K and Hirano A: Hirano bodies and Alzheimer's disease. Kaohsiung J Med Sci 13: 10-18, 1997.

62. Connor B, Beilharz EJ, Williams C, Synek B, Gluckman PD, Faull RL and Dragunow M: Insulin-like growth factor-I (IGF-I) immunoreactivity in the Alzheimer's disease temporal cortex and hippocampus. Brain Res Mol Brain Res 49: 283-290, 1997.

63. Connor B, Young D, Yan Q, Faull RL, Synek B and Dragunow M: Brain-derived neurotrophic factor is reduced in Alzheimer's disease. Brain Res Mol Brain Res 49: 71-81, 1997.

64. Griffith CM, Eid T, Rose GM and Patrylo PR: Evidence for altered insulin receptor signaling in Alzheimer's disease. Neuropharmacology 136: 202-215, 2018. 